\title{
Lidil
}

Revue de linguistique et de didactique des langues

$63 \mid 2021$

Littératie numérique et didactique des langues et des cultures

\section{Outils d'aide à la traduction : pratiques numériques ordinaires en contexte scolaire}

Translation Tools: Ordinary Digital Practices in a School Context

\section{Aurélie Bourdais}

\section{OpenEdition}

\section{Journals}

Édition électronique

URL : http://journals.openedition.org/lidil/8819

DOI : $10.4000 /$ lidil.8819

ISSN : 1960-6052

Éditeur

UGA Éditions/Université Grenoble Alpes

Édition imprimée

ISBN : 978-2-37747-283-3

ISSN : $1146-6480$

Référence électronique

Aurélie Bourdais, «Outils d'aide à la traduction : pratiques numériques ordinaires en contexte scolaire », Lidil [En ligne], 63 | 2021, mis en ligne le 30 avril 2021, consulté le 28 avril 2021. URL : http:// journals.openedition.org/lidil/8819; DOI : https://doi.org/10.4000/lidil.8819

Ce document a été généré automatiquement le 28 avril 2021.

(c) Lidil 


\title{
Outils d'aide à la traduction : pratiques numériques ordinaires en contexte scolaire
}

Translation Tools: Ordinary Digital Practices in a School Context

\author{
Aurélie Bourdais
}

\section{Introduction}

1 Bien que les outils d'aide à la traduction n'aient pas été initialement conçus à destination d'un public scolaire, les élèves semblent s'en être emparés depuis quelques années. Selon une enquête réalisée en lycée en 2017 auprès de 118 lycéens, ils seraient plus de $60 \%$ à avoir recours à WordReference, et plus de $90 \%$ à consulter Google Traduction dans le cadre d'activités scolaires (Bourdais \& Guichon, 2020), en dépit des interdictions d'une majorité d'enseignants (Ducar \& Schocket, 2018; Valijärvi \& Tarsoly, 2019). Le recours à ces outils, qui seraient souvent consultés dans le cadre de devoirs à la maison, se situe à la croisée de la littéracie scolaire et des pratiques numériques personnelles et souligne le lien fort entre l'école et l'écrit. Il semble aujourd'hui difficile d'ignorer ces pratiques qui soulèvent des questions didactiques légitimes.

2 L'étude de cas présentée ici vise à analyser les pratiques numériques personnelles d'une lycéenne dans une perspective exploratoire. Après avoir établi le lien entre outils d'aide à la traduction et littéracie numérique, l'article s'attachera à identifier les savoirs et savoir-faire mis en œuvre par la lycéenne lors de la consultation de ces outils et plus spécifiquement d'un traducteur automatique et à identifier les liens entre pratiques personnelles et pratiques scolaires. 


\section{Outils en ligne et littéracies numériques}

3 La récurrence du terme de littéracie pour décrire des compétences et des pratiques numériques (Bawden, 2008; Gerbault, 2012; Lacelle \& Lebrun, 2014; Leu, 2000 ; Selwyn, 2009) nous invite à définir cette notion « déictique » (Leu et coll., 2013), avant de réfléchir aux implications du choix de ce terme associé au numérique. Nous considèrerons enfin la consultation des outils d'aide à la traduction en tant que pratique littéracique en contexte scolaire.

\subsection{De la littéracie aux pratiques littéraciques}

4 La notion de «littéracie » est historiquement liée aux travaux de Goody, qui s'est intéressé aux « conséquences de l'écriture » dès les années 1960 (Goody \& Watt, 1963) : le développement de l'écriture a d'une part joué un rôle mnémonique déterminant et a, d'autre part, favorisé le développement d'une réflexivité sur la langue. En modifiant nos modes de pensée, le développement de l'écrit a eu des répercussions majeures sur l'organisation des sociétés (Goody, 1977, 2006).

5 Bien que Goody se soit défendu de tout « déterminisme technologique » (Goody, 2006, p. 10), on lui a reproché d'attribuer à la littéracie des effets uniformes, indépendants de tout contexte socioculturel (Scribner \& Cole, 2010). Les New Literacy Studies présentent au contraire la littéracie comme des pratiques sociales, situées et plurielles (Barton \& Hamilton, 2010 ; Street, 1997). L'école constitue un contexte social qui entraine la mise en œuvre de pratiques littéraciques distinctes des pratiques extrascolaires, ce dont collégiens et lycéens ont conscience (Barré-De Miniac, 2001).

6 Loin d'être exclusives, ces deux conceptions de la littéracie sont complémentaires. Ainsi, Jaffré définit la littéracie comme :

[...] l'ensemble des activités humaines qui impliquent l'usage de l'écriture, en réception et en production. Elle met un ensemble de compétences de base, linguistiques et graphiques, au service de pratiques, qu'elles soient techniques, cognitives, sociales ou culturelles. Son contexte fonctionnel peut varier d'un pays à

l'autre, d'une culture à l'autre, et aussi dans le temps. (2004, p. 31).

7 En plaçant au cœur de la notion les pratiques en tant qu'actualisation de compétences et en rappelant leur nature située, Jaffré dépasse la dichotomie entre approche cognitive et approche sociale. En dépit de ses spécificités techniques, la littéracie numérique se situe dans le prolongement de la littéracie traditionnelle.

\subsection{La littéracie numérique : compétences et pratiques}

8 L'emploi du terme de littéracie dans un contexte numérique rappelle le lien étroit entre numérique et écrit (Daunay \& Fluckiger, 2018). La littéracie numérique a tout d'abord fait référence à des compétences variées : des compétences informationnelles (Bawden, 2008; Lacelle \& Lebrun, 2014), techniques, comme la sélection des outils disponibles (Ollivier, 2018) ou sémiotiques (Lebrun et coll., 2013). Ces compétences ne sont pas nouvelles mais se complexifient face à la profusion de sources et d'informations immédiatement disponibles (Leu et coll., 2013). La littéracie numérique ne saurait toutefois se réduire à une multiplicité de compétences. 
9 De même que la littéracie traditionnelle, la littéracie numérique renvoie à des pratiques sociales (Daunay \& Fluckiger, 2018). Les compétences évoquées doivent alors être mises en œuvre "dans un cadre critique et éthique », au sein duquel «l'usager [a] conscience du comportement approprié lié à une situation et un environnement numérique spécifiques" (Ollivier, 2018, p.13). Dans le domaine de l'éducation, Fluckiger rappelle la nécessité de considérer les pratiques personnelles des adolescents dans leur pluralité (2016) et de ne pas surestimer les effets de la technologie au détriment du rôle des acteurs (2019). L'exemple des outils d'aide à la traduction suggère que les pratiques littéraciques, numériques ou non, ne sont pas toutes valorisées socialement de la même façon.

\subsection{Outils d'aide à la traduction et pratiques littéraciques scolaires}

Les outils d'aide à la traduction sont nombreux et complexes. Je présenterai une brève typologie des trois principaux outils consultés par les élèves dans le cadre de mes observations. Par souci de clarté, je les identifierai selon les fonctionnalités utilisées par les élèves.

11 WordReference est un "ensemble de dictionnaires bilingues et unilingues " qui mobilise une équipe de linguistes et de programmateurs (Crespi, 2017, p. 106). L'affranchissement du support papier combiné à l'immense capacité de stockage en ligne (L'Homme \& Cormier, 2014) a permis à WordReference de proposer les traductions de nombreuses collocations pour le même terme. L'utilisateur choisit la traduction adéquate selon la nature du mot et le contexte.

12 Google Traduction est un traducteur automatique : il analyse un corpus constitué de textes bilingues traduits humainement et effectue des calculs de probabilités pour proposer une traduction unique (Poibeau, 2019). Les chances d'obtenir une traduction linguistiquement correcte varient en fonction de la contextualisation de la recherche et de l'importance du corpus de textes déjà traduits entre les deux langues (Groves \& Mundt, 2015 ; Poibeau, 2019). Il revient ensuite à l'utilisateur de vérifier la validité de la proposition.

13 Enfin, Linguee est un concordancier : il s'appuie sur un corpus similaire à celui analysé par les traducteurs en ligne. Mais au lieu d'effectuer des calculs statistiques, il propose une sélection d'extraits de corpus à l'utilisateur, qui choisit la traduction appropriée en fonction du contexte.

14 Les lycéens auraient massivement recours aux outils d'aide à la traduction à des fins de génération de texte et de révision (Bourdais \& Guichon, 2020), c'est-à-dire dans le cadre du processus rédactionnel tel qu'il a été modélisé par Flower et Hayes (1981). Ces outils sont souvent consultés dans le cadre des devoirs faits à la maison, c'est-à-dire dans le cadre de pratiques littéraciques "scolaires ", que Fluckiger (2008, p. 59) distingue des pratiques « ordinaires » des élèves. Les premières se caractérisent par une imposition de normes et de modèles qui peut entrer en tension avec les compétences du scripteur et être génératrice d'« insécurité scripturale» (Dabène, 1991, p. 14), voire d'échec scolaire (Lahire, 1993). Selon Penloup (2012), les pratiques scolaires relèvent des " pratiques dominantes de la littéracie », et s'opposent aux « littéracies vernaculaires » (p. 2). Elle reprend ici la distinction effectuée par Barton et Hamilton (2010), selon qui « [1]es pratiques de l'écrit sont modelées par les institutions sociales et les relations de pouvoir, et certaines littéracies sont plus dominantes, plus visibles et plus influentes 
que d'autres » (p. 2). Il n'est pas impossible que les préconisations des enseignants, qui interdiraient Google Traduction et recommanderaient WordReference, soient non seulement liées à des questions didactiques mais qu'elles soient aussi motivées par certaines représentations de ces outils : le dictionnaire serait considéré comme plus légitime car relevant d'une littéracie "dominante», tandis que Google Traduction serait placé du côté des littéracies "vernaculaires", "ancrées dans l'expérience quotidienne, [...] souvent peu valorisées par la société et [qui] ne sont pas particulièrement soutenues ni régulées par des institutions sociales externes » (ibid., p. 58-59).

Ces pratiques "buissonnières", où les élèves peuvent révéler une "créativité dispersée, tactique et bricoleuse " (Certeau, 1990, p. XL) s'immiscent dans les pratiques scolaires. Ce télescopage entre sphère privée et sphère académique, cette intercontextualité (Guichon, 2015) soulève des questions fondamentales sur les relations complexes entre l'école et le monde extérieur (Daunay \& Fluckiger, 2018; Maulini \& Perrenoud, 2005). La reconnaissance des pratiques ordinaires des élèves pourrait alors constituer un enjeu pour l'enseignant, dont le rôle serait de «faire passer [l'élève] de la "connaissance" intériorisée et implicite à un "savoir" qui résulte, lui, d'un processus d'objectivation de la connaissance et de sa théorisation dans un langage approprié, explicite et, dès lors transférable » (Penloup, 2012, p. 6) et de l'accompagner dans le développement d'un rapport réflexif aux pratiques numériques (Daunay \& Fluckiger, 2018).

16 L'étude exploratoire présentée ici vise à analyser les pratiques numériques personnelles d'une lycéenne pour (1) identifier les savoirs et savoir-faire mis en œuvre dans le cadre de la consultation des outils d'aide à la traduction en contexte scolaire et (2) déterminer dans quelle mesure ces savoirs et savoir-faire sont liés aux pratiques littéraciques scolaires.

\section{Méthodologie}

17 Les études de cas offrent un cadre de recherche privilégié pour observer les singularités propres à chacun dans une approche holistique (Duff, 2012, p.98). Ce dispositif longitudinal semble approprié pour observer les pratiques numériques personnelles de lycéens en prenant en compte leur dimension cognitive et sociale.

\subsection{Présentation du terrain de recherche et des participants}

Cette étude s'appuie sur une expérimentation menée dans un lycée général et technologique de l'agglomération lyonnaise, dans le cadre d'un cours d'anglais de langue vivante approfondie (LVA). Elle a impliqué les 13 élèves de terminale littéraire (TL) pour qui cet enseignement de spécialité s'ajoutait à l'enseignement d'anglais commun à tous les élèves et à celui de littérature étrangère en anglais, obligatoire en série L, ce qui représentait un enseignement cumulé de 6,5 h d'anglais hebdomadaire. Les élèves de ce groupe, curieux, sérieux et travailleurs selon leur enseignante, ont préparé des épreuves du baccalauréat visant à évaluer le niveau C1 dans les compétences écrites et orales. 


\subsection{Protocole de recherche}

L'expérimentation s'est déroulée sur trois séances (S1, S2 et S3) selon les modalités décrites ci-dessous (tableau 1). Une séance préliminaire a été organisée une semaine avant la S1 pour présenter le cadre juridique et le formulaire de consentement, et pour guider les élèves volontaires dans l'installation de l'application X-Recorder, utilisée pour les captures d'écran dynamiques (S1 et S2), sur leur téléphone portable.

Tableau 1. - Protocole de recherche : consignes, objectifs et données recueillies.

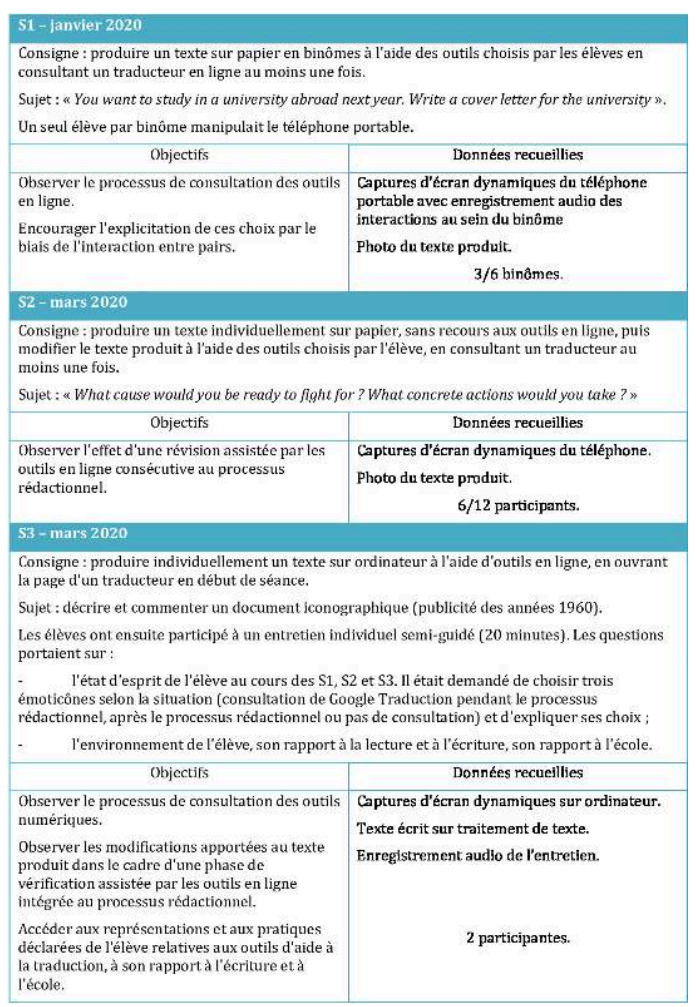

L'ensemble des élèves présents ont participé aux S1 et S2, qui se sont déroulées pendant un cours d'anglais. L'enseignante, absente pendant la S1, était présente pendant la S2. La S3 a été organisée au lycée, sur le temps libre des élèves. Les chiffres dans le tableau 1 indiquent le nombre de données recueillies par rapport au nombre d'élèves présents. L'écart important entre ces deux chiffres s'explique par le fait que quelques élèves ne souhaitaient pas réaliser de capture d'écran de leur téléphone, d'autres ont rencontré des difficultés pour partager le fichier vidéo en raison de difficultés de connexion à Internet dans l'établissement. Enfin, quatre entretiens avaient été prévus, mais le protocole a été interrompu en raison de la crise sanitaire.

\subsection{Analyse des données}

21 Les captures d'écran ont donné lieu à une analyse descriptive des indicateurs suivants :

- sélection de l'outil ;

- type de texte saisi (texte-source) : mot, groupe de mots, phrase, paragraphe ;

- traitement de la traduction : modification du texte-source, consultation d'autres outils. 
Tableau 2. - Outils consultés pendant les 3 séances.

\begin{tabular}{|c|c|c|c|c|c|}
\hline & Linguee & $\begin{array}{l}\text { Google } \\
\text { Traduction }\end{array}$ & Wordreference & Autres & TOTAL \\
\hline $\begin{array}{l}\text { S1 } \\
3 \text { binôm }\end{array}$ & 0 & 18 & 13 & 7 & 38 \\
\hline $\begin{array}{l}\text { S2 } \\
6 \text { élèves }\end{array}$ & 12 & 16 & 35 & 0 & 63 \\
\hline $\begin{array}{l}\text { S3 } \\
2 \text { élèves }\end{array}$ & 0 & 20 & 0 & 0 & 20 \\
\hline TOTAL & 12 & 54 & 48 & 7 & 121 \\
\hline
\end{tabular}

Compte tenu du nombre restreint de participants, ces données ne peuvent être considérées comme représentatives. Elles sont plutôt en accord avec les pratiques déclarées des lycéens en ce qui concerne le recours à WordReference et à Google Traduction, mais on peut noter que Reverso n'a pas été consulté (Bourdais \& Guichon, 2020).

\subsection{Choix de la participante}

Des données complètes ont pu être recueillies pour deux lycéennes, Raphaëlle et Elsa ${ }^{1}$. Elsa est une élève qui justifie son choix de suivre l'enseignement de LVA par ses compétences et par son goût pour l'anglais. Elle ne se présente toutefois ni comme excellente ( «j'ai quelques facilités»), ni comme passionnée («j'aime bien l'anglais »), contrairement à Raphaëlle, qui s'oriente vers des études d'anglais pour devenir traductrice. Le choix de présenter Elsa dans le cadre de cette étude de cas est motivé par la plus grande richesse des données recueillies, en particulier en ce qui concerne la consultation de Google Traduction. 


\section{Résultats : des pratiques inventives et intuitives}

Bien que les outils consultés par Elsa varient selon les séances, la jeune fille suit globalement la tendance du groupe en consultant surtout Google Traduction et WordReference (tableau 3). Après avoir identifié le type de recherches effectuées selon les outils, nous analyserons le processus de consultation des traducteurs et l'utilisation des traductions obtenues.

\subsection{Sélection de la ressource : des pratiques «ignorées »}

Après avoir analysé les pratiques effectives d'Elsa, je confronterai celles-ci au mode opératoire qu'elle décrit lors de l'entretien afin de déterminer dans quelle mesure Elsa parvient à rendre compte des compétences qu'elle met en œuvre lorsqu'elle consulte les outils en ligne.

\subsubsection{Une sélection pertinente des outils d'aide à la traduction}

Les recherches effectuées par Elsa au cours des trois séances ont été comptabilisées en fonction de l'outil consulté et du type de texte saisi (tableau 3).

Tableau 3. - Type de recherche effectuée selon l'outil.

\begin{tabular}{ccccc}
\hline & $\begin{array}{c}\text { Google } \\
\text { Traduction }\end{array}$ & $\begin{array}{c}\text { Google (moteur } \\
\text { de recherche) }\end{array}$ & Wordreference & $\begin{array}{c}\text { Macmillan } \\
\text { dictionary }\end{array}$ \\
\hline Unités lexicales & 4 & 1 & $\mathbf{1 0}$ & 0 \\
\hline Groupe de mots & $\mathbf{1 2}$ & 4 & 0 & 1 \\
\hline Total & 16 & 5 & 10 & 1 \\
\hline
\end{tabular}

Elsa a consulté quatre outils de façon diversifiée selon les séances: elle a consulté exclusivement Google Traduction et WordReference lorsqu'elle a travaillé seule (S2 et S3), le dictionnaire Macmillan et le moteur de recherche Google ont été sollicités uniquement pendant la S1, à l'initiative d'Alice, la lycéenne qui travaillait en binôme avec Elsa.

31 Les résultats montrent qu'Elsa consulte WordReference pour traduire exclusivement des unités lexicales, que Bogaards (1994) définit comme les plus petites parties du discours constituées d'au moins un constituant sémantique et d'au moins un mot, et privilégie le traducteur en ligne pour traduire des groupes nominaux, des groupes verbaux ou des phrases ( 12 recherches contre 4 portant sur des unités lexicales ${ }^{2}$ ). Elsa consulte d'abord Google Traduction pour traduire le syntagme nominal " être vivant ", mais lorsque le traducteur lui propose un groupe verbal, elle sollicite le dictionnaire. La lycéenne semble donc avoir compris à la fois la notion d'unité lexicale et le fonctionnement de WordReference et de Google Traduction. Les propos tenus par Elsa lors de l'entretien (S3) laissent toutefois penser qu'il s'agit de connaissances implicites. 


\subsubsection{Un décalage entre pratiques déclarées et pratiques observées} traducteurs automatiques :

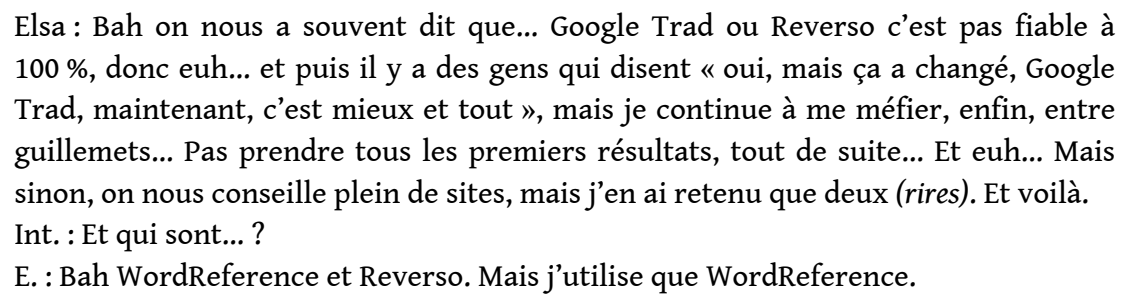

Face à une profusion de conseils parfois contradictoires, Elsa semble avoir adopté une ligne de conduite claire et ne consulter que le dictionnaire en ligne. Néanmoins, les choses sont moins simples qu'il n'y parait lorsqu'Elsa décrit sa méthode de façon plus détaillée :

Elsa : des fois, j'essaie de taper des phrases, ou des mots, enfin des expressions, et euh, ça me met pas, enfin... c'est pas ce que je recherche, soit ça va pas avec la phrase, soit c'est pas le bon sens... ou quoi, mais des fois c'est un peu galère, euh, des fois, de trouver la bonne expression. Et euh...

Int. : Et qu'est-ce que tu fais, alors, quand tu as l'impression que c'est pas le bon sens, ou que ça correspond pas à ce que tu recherches?

E. : Euh... Je vais sur d'autres sites ou sur d'autres traducteurs. Enfin, plus un dictionnaire, parce que je connais pas trop les traducteurs, du coup (rire). [...] Ou alors je tape directement le... le mot que... en anglais ou en français dont j'ai besoin, sur Internet, comme ça, pas sur un site, et euh... je peux trouver des trucs, des fois. Int. : D'accord. Dans le moteur de recherche, c'est ça?

E. : Voilà.

Int. : D'accord. Donc, tu tapes le mot en français, et puis... comment tu as la réponse, alors ? [...]

E. : Bah j'écris euh... Enfin des fois, ça marche pas trop euh... En français euh... Du français-anglais. Mais par exemple, anglais-français ça peut marcher parfois de... d'écrire le mot... et après mettre euh, « traduction ».

propos d'Elsa sont confus, la jeune fille éprouve des difficultés à exposer son mode opératoire. Elle reconnait aussi ne pas faire de différence entre traducteur et dictionnaire. Cette prise de conscience a eu lieu au début de la S3, lorsqu'Elsa a ouvert WordReference alors qu'il lui avait été demandé d'ouvrir la page d'un traducteur en ligne. Je lui ai indiqué qu'il ne s'agissait pas d'un traducteur mais d'un dictionnaire. Elsa a alors ouvert Google Traduction et n'a pas consulté d'autre outil.

Les pratiques déclarées d'Elsa sont en adéquation avec celles observées lors de la S1, où le moteur de recherche est sollicité plusieurs fois à l'initiative d'Alice, mais pas lorsqu'elle travaille seule (S2 et S3). Il est alors possible que la confusion dans le discours de la participante soit liée au fait qu'elle tente de décrire une pratique qui n'est pas la sienne, à moins que les captures d'écran $\mathrm{S} 2$ et $\mathrm{S} 3$ ne donnent accès à des pratiques situées et peu représentatives.

Les captures d'écran montrent qu'en dépit d'un discours hésitant, Elsa a compris la différence fondamentale entre un dictionnaire en ligne, qui apporte une aide lexicale, et un traducteur en ligne, où la contextualisation est souvent essentielle. Ce décalage entre pratiques déclarées et pratiques réelles fait écho aux « connaissances ignorées » mentionnées par Penloup (2012) : peut-être qu'Elsa n'a pas conscience de ses pratiques, mais il est aussi possible qu'elle ne mentionne pas son utilisation de Google Traduction 
car elle ne prête aucune légitimité à cet outil dans un contexte scolaire. Les captures d'écran révèlent que, lorsqu'Elsa consulte le traducteur en ligne, elle a développé certains "arts de faire» (Certeau, 1990) pour évaluer la validité des traductions proposées et pour les utiliser.

\subsection{Traitement des traductions : des « arts de faire »}

Les pratiques d'Elsa relatives à WordReference sont en adéquation avec celles du groupe : sur les 48 consultations du dictionnaire observées, 40 concernent des unités lexicales et le traitement de la traduction n'est généralement pas problématique. L'homogénéité des pratiques observées pourrait s'expliquer par le fait que près de la moitié des enseignants apporterait une aide méthodologique pour utiliser le dictionnaire en ligne (Bourdais \& Guichon, 2020). Au contraire, les traducteurs automatiques restent généralement des outils clandestins, que les élèves semblent découvrir en dehors du contexte scolaire. Je m'intéresserai ici à certains «arts de faire» mis en œuvre par Elsa lors de l'évaluation et de l'utilisation des traductions proposées par Google Traduction.

\subsubsection{Vérification des traductions proposées par Google Traduction}

L'une des spécificités du traducteur en ligne tel qu'il est utilisé par les lycéens est de proposer une traduction unique, mais celle-ci n'est pas nécessairement correcte. Il revient alors à l'utilisateur d'évaluer la traduction. Lorsqu'Elsa doute de la validité de la traduction proposée, on peut observer deux types de comportements :

1. Elsa effectue un travail de pré-édition : lorsqu'elle cherche la traduction du mot "marque » et qu'elle n'est pas certaine que la proposition («mark») soit appropriée, elle ajoute un complément au nom du texte-source : « marque de chaussure », ce qui lève l'ambigüité due à la polysémie du mot ;

2. Elsa consulte un autre outil en ligne : lorsque Google Traduction traduit « être vivant » par «be alive », Elsa sollicite WordReference, où elle trouve la traduction recherchée : «living being ".

Ce recours à WordReference n'est observé que lorsque les recherches portent sur des unités lexicales. Elsa a compris que le dictionnaire n'était pas un outil adapté pour traduire des segments plus longs. Dans ce cas, elle consulte Google Traduction.

\subsubsection{Utilisation des traductions proposées par Google Traduction}

40 L'utilisation qu'Elsa fait des traductions obtenues par le biais du traducteur en ligne est présentée dans le tableau suivant (tableau 4). Le texte saisi par Elsa figure dans la colonne correspondant à la façon dont elle utilise la traduction proposée : soit elle la reproduit à l'identique, soit elle y apporte des modifications d'ordre grammatical ou sémantique, soit elle effectue une nouvelle recherche en modifiant le texte-source. 
Tableau 4. - Utilisation des traductions obtenues avec Google Traduction - S3.

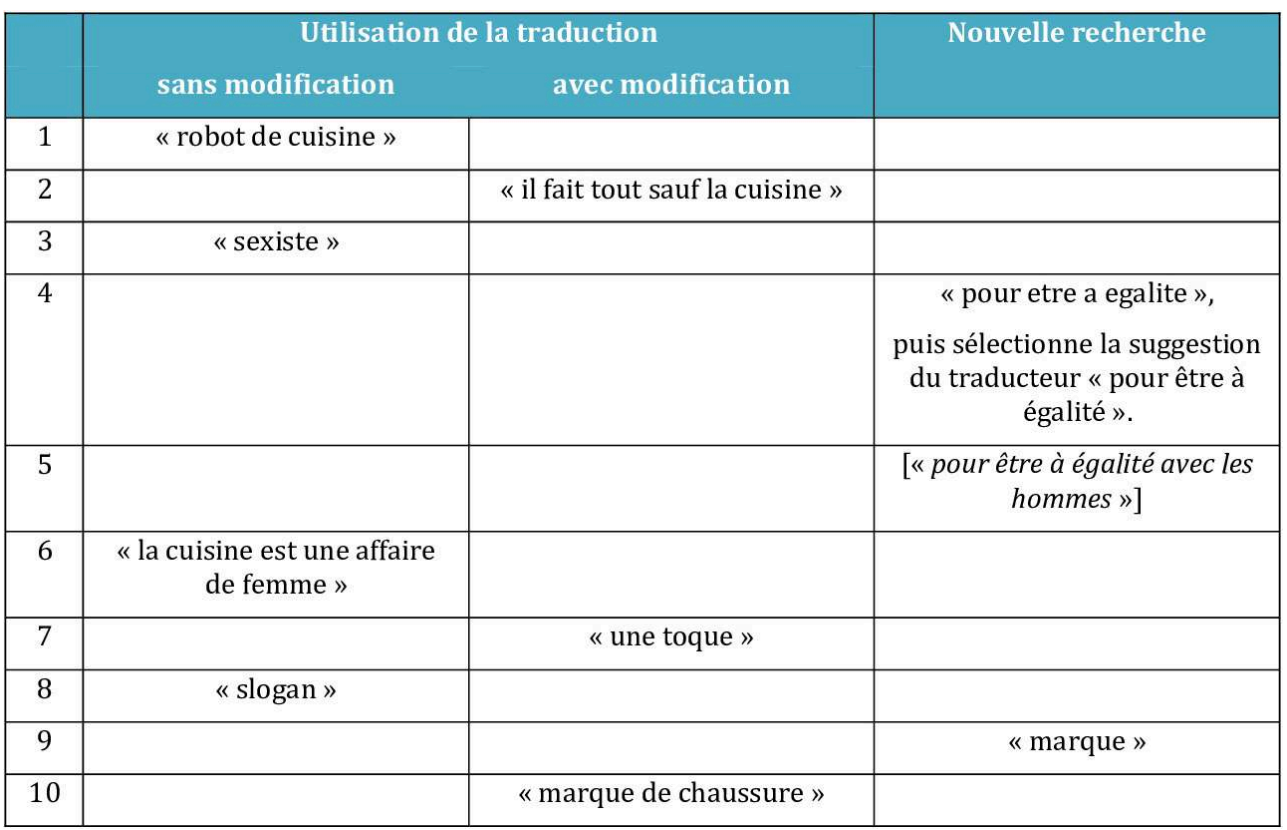

Elsa a utilisé la majorité des traductions obtenues, avec modification (items 2, 7 et 10) ou sans (items 1, 3, 6 et 8). Deux autres propositions ont entrainé une nouvelle recherche (items 4 et 9). Enfin, une traduction, indiquée entre crochets, n'a pas été utilisée (item 5). La reconnaissance semble jouer un rôle essentiel dans l'utilisation de la traduction: "sexist ", "slogan " sont des mots transparents et "kitchen robot " est aisément compréhensible, contrairement à la traduction "to be on par with men", qu'Elsa n'emploie pas. Le comportement d'Elsa est ici conforme à ses déclarations : face à une expression qui ne lui évoque rien, la jeune fille se méfie et emploie une expression qui lui est familière.

Les modifications apportées aux traductions relèvent de la post-édition et ont des fonctions diverses :

1. Apporter une précision sémantique : Elsa transforme le nom « hat » en nom composé («cook hat ») ;

2. Corriger une erreur morphosyntaxique causée par l'ambigüité du texte saisi : Elsa remplace le pronom « he » par le groupe nominal « the chef », qui renvoie à un objet ;

3. Employer seulement la partie pertinente de la traduction : Elsa emploie le nom «brand » car le complément « de chaussure » avait uniquement une fonction contextualisante.

Elsa semble ainsi avoir établi envers Google Traduction une distance critique qui, combinée à ses compétences linguistiques, lui permet d'utiliser le traducteur avec une certaine efficacité.

\section{Discussion}

Malgré sa méconnaissance des différences entre traducteurs et dictionnaires, Elsa sélectionne WordReference ou Google Traduction avec pertinence, privilégiant l'un pour traduire des segments grammaticaux et l'autre pour des unités lexicales, et se livre à un travail de pré-édition ou de post-édition lorsqu'elle doute de la proposition du traducteur. Ces activités de révision, incluses dans le processus de consultation des 
traducteurs s'ajoutent aux activités de révision du texte incluses dans le processus rédactionnel (Flower \& Hayes, 1981). Ces pratiques semblent s'être développées de façon intuitive, du moins ne sont-elles pas verbalisées. Elles n'en sont pas moins efficaces.

La difficulté d'Elsa, sa réticence, peut-être, à reconnaitre qu'elle utilise Google Traduction suggère qu'elle a, pour reprendre les termes de Dabène (1991, p. 9), «intériorisé les frontières» entre des pratiques littéraciques scolaires, comme la consultation de dictionnaires, et des pratiques ordinaires, comme le recours à Google Traduction, qui n'auraient pas leur place dans un contexte scolaire et encore moins dans un enseignement d'anglais de spécialité. Les pratiques observées semblent toutefois contredire ces représentations. En effet, c'est en s'appuyant sur des connaissances linguistiques scolaires (la polysémie des mots, les natures grammaticales) qu'Elsa parvient à développer un usage informé du traducteur en ligne et à obtenir des traductions correctes. Pratiques ordinaires et pratiques scolaires ne s'opposent donc pas, elles semblent se combiner dans une intercontextualité qui permet à Elsa d'utiliser ces différents outils de façon fluide.

La méfiance de la lycéenne envers le traducteur semble l'inciter à utiliser des traductions dont elle peut elle-même évaluer la validité de façon immédiate. Elle ne semble pas considérer Google Traduction comme un outil favorisant l'enrichissement lexical. L'exemple de l'expression qu'elle n'a pas utilisée (" to be on a par with men ») laisse toutefois entrevoir des exploitations didactiques possibles, par le biais d'activités visant à inciter les lycéens à consulter d'autres ressources comme WordReference, pour vérifier le sens d'un mot inconnu. Le traducteur en ligne pourrait alors constituer une aide à l'enrichissement lexical, ainsi qu'à la vérification de la grammaticalité d'une phrase.

L'étude menée souligne les difficultés multiples pour accéder aux pratiques numériques effectives des élèves. Des difficultés techniques ont été rencontrées. Une minorité d'élèves sont parvenus à réaliser une capture d'écran et à partager leurs vidéos, en raison de difficultés de connexion à Internet propres au lycée et peut-être aussi d'une certaine hétérogénéité des compétences numériques des adolescents (Fluckiger, 2011). Mais les difficultés sont surtout liées à la nature même de l'objet de recherche et au mode d'observation: quelques élèves ont refusé de participer à l'expérimentation car ils ont perçu la captation de leur écran de téléphone portable comme intrusive. L'observation de pratiques vernaculaires, perçues comme illégitimes, dans un contexte scolaire, a également entrainé des résistances. Enfin, le protocole a pu biaiser le comportement des élèves : le recours exclusif d'Elsa à Google Traduction (S3) n'est en adéquation ni avec ses pratiques déclarées, ni avec les pratiques observées lors de la S2 et s'explique probablement par mon intervention. Les enjeux scientifiques de la recherche menée sont alors entrés en conflit avec le souci d'une approche écologique. Les données recueillies donnent à voir des pratiques plurielles et diversifiées, ce qui pourrait laisser penser que leur représentativité est problématique. Il est toutefois possible que cette pluralité des pratiques soit constitutive du comportement de l'individu, dont les pratiques varient selon les contextes d'usage (Fluckiger, 2016). 


\section{Conclusion}

La consultation des outils d'aide à la traduction met en œuvre des compétences linguistiques et techniques en contexte scolaire. En ce sens, elle relève d'une pratique littéracique numérique. La méfiance d'Elsa ainsi que les résistances exprimées par certains élèves envers les traducteurs en ligne suggèrent toutefois que le recours à cet outil relève de pratiques vernaculaires, considérées comme peu légitimes. La consultation de Google Traduction semble donc se situer dans un entre-deux, ou, pour reprendre les termes de Guichon(2015), dans une intercontextualité où pratiques personnelles et pratiques scolaires s'entremêlent.

L'étude de cas présentée ici n'a pas de visée généralisante mais donne accès à des pratiques singulières. Elle donne à voir des stratégies de vérification efficaces mises en œuvre par une élève qui suit un enseignement de spécialité en anglais, mais les pratiques observées au sein du groupe, et en particulier auprès de Raphaëlle, laissent penser que les compétences linguistiques ne sont pas suffisantes pour développer des compétences dans la consultation des outils d'aide à la traduction. D'autres recherches impliquant des élèves non spécialistes seraient nécessaires pour mieux comprendre les liens entre compétences linguistiques et développement de stratégies efficaces. L'étude menée ne permet pas non plus de déterminer dans quelle mesure Elsa serait capable de rendre compte de ses pratiques en s'appuyant sur des connaissances métalinguistiques. L'organisation d'auto-confrontations pourrait permettre de répondre à cette question. Les pratiques observées laissent penser qu'Elsa en a au moins des connaissances intuitives. Il semble que l'explicitation de ces pratiques, leur verbalisation aurait toute sa place dans un cours de langue, où le partage de connaissances et la construction de compétences dans une approche collaborative pourraient être bénéfiques à l'ensemble des élèves.

\section{BIBLIOGRAPHIE}

BARRÉ-DE MINIAC, Christine (2001). De l'école au lycée : liens ou ruptures entre les pratiques extrascolaires et les pratiques scolaires d'écriture ? Repères, 23(1), 93-109. <https://doi.org/ 10.3406/reper.2001.2359>.

BARTON, David \& HAMILTON, Mary. (2010). La littératie : une pratique sociale. Langage et société, 133(3), 45-62.

BAWDEN, David. (2008). Origins and Concepts of Digital Literacy. Dans C. Lankshear \& M. Knobel (dir.), Digital Literacies. Concepts, Policies and Practices (p. 17-32). Peter Lang.

BOGAARDS, Paul. (1994). Le vocabulaire dans l'apprentissage des langues étrangères. Didier.

BOURDAIS, Aurélie \& GUICHON, Nicolas. (2020). Représentations et usages du traducteur en ligne par les lycéens. Alsic, 23(1). <https://doi.org/10.4000/alsic.4533>.

CERTEAU, Michel DE. (1990). L'invention du quotidien. Vol. 1 : Arts de faire (1 ${ }^{\text {re }}$ éd. 1980). Gallimard. 
CRESPI, Patrizia. (2017). WordReference : une Wikipédia lexicographique. Revue française de linguistique appliquée, 22(1), 103-115.

DABÈNE, Michel. (1991). Un modèle didactique de la compétence scripturale. Repères, 4(1), 9-22. <https://doi.org/10.3406/reper.1991.2030>.

DAUNAY, Bertrand \& FLUCKIGER, Cédric. (2018). Écriture et numérique : pourquoi et comment parler de littéracie numérique ? Recherches, 69, 71-86.

DuCAR, Cynthia \& SCHоскеT, Deborah Houk. (2018). Machine Translation and the L2 Classroom: Pedagogical Solutions for Making Peace with Google Translate. Foreign Language Annals, 51(4), 779-795. <https://doi.org/10.1111/flan.12366>.

Duff, Patricia. (2012). How to Carry out Case Study Research. Dans A. Mackey \& S. M. Gass (dir.), Research Methods in Second Language Acquisition (p. 95-116). John Wiley \& Sons. <https://doi.org/ 10.1002/9781444347340.ch6>.

FLOWER, Linda \& HAYES, John. (1981). A Cognitive Process Theory of Writing. College Composition and Communication, 32(4), 365-387. <https://doi.org/10.2307/356600>.

FLUCKIGER, Cédric. (2008). L'école à l'épreuve de la culture numérique des élèves. Revue française de pédagogie, 163, 51-61. <https://doi.org/10.4000/rfp.978>.

FLUCKIGER, Cédric. (2011). La didactique de l'informatique et les constructions sociales de la figure des jeunes utilisateurs. Recherches en didactiques, 11(1), 67-83.

FLUCKIGER, Cédric. (2016). Culture numérique, culture scolaire : homogénéités, continuités et ruptures. Diversité, 185, 64-70.

FLUCKIGER, Cédric. (2019). Numérique en formation : des mythes aux approches critiques. Éducation permanente, 219, 17-30.

GERBAULT, Jeannine. (2012). Littératie numérique. Les nouvelles dimensions de l'écrit au $21^{\mathrm{e}}$ siècle. Recherches en didactique des langues et des cultures, 9(2). <https://doi.org/10.4000/rdlc.3960>.

Goody, Jack. (1977). The Domestication of the Savage Mind. Cambridge University Press.

Goody, Jack. (2006). La technologie de l'intellect (J.-C. Lejosne, trad.). Pratiques, 131(1), 7-30. (Texte original publié en 1968 en introduction à Literacy in Traditional Societies. Cambridge University Press). <https://doi.org/10.3406/prati.2006.2114>.

GooDY, Jack \& WATT, Ian. (1963). The Consequences of Literacy. Comparative Studies in Society and History, 5(3), 304-345.

GRoves, Michael \& MUNDT, Klaus. (2015). Friend or Foe? Google Translate in Language for Academic Purposes. English for Specific Purposes, 37, 112-121. <https://doi.org/10.1016/j.esp. 2014.09.001>.

GuICHON, Nicolas. (2015). Quelle transition numérique pour les étudiants internationaux ? Alsic, 18(1). <https://doi.org/10.4000/alsic.2793>.

JAFFRÉ, Jean-Pierre. (2004). La litéracie : histoire d'un mot, effets d'un concept. Dans C. BarréDe Miniac, C. Brissaud \& M. Rispail (dir.), La littéracie. Conceptions théoriques et pratiques d'enseignement de la lecture-écriture (p. 21-41). L'Harmattan.

LACELLE, Nathalie \& LEBRUN, Monique. (2014). La littératie médiatique multimodale : réflexions sémiologiques et dispositifs concrets d'application. Forumlecture.ch, 2, 1-17. 
LAHIRE, Bernard. (1993). Culture écrite et inégalités scolaires : sociologie de "l'échec scolaire » à l'école primaire. Presses universitaires de Lyon.

LEBRUN, Monique, LACELLE, Nathalie \& Boutin, Jean-François. (2013). La littératie médiatique à l'école : une (r)évolution multimodale. Globe, 16(1), 71-89. <https://doi.org/10.7202/1018178ar>.

LEU, Donald. (2000). Our Children's Future: Changing the Focus of Literacy and Literacy Instruction. The Reading Teacher, 53(5), 424-429.

Leu, Donald, KInzer, Charles, CoIro, Julie, CASTEK, Jill \& Henry, Laurie. (2013). New Literacies : A Dual-Level Theory of the Changing Nature of Literacy, Instruction, and Assessment. Dans R. Ruddel \& N. Unrau (dir.), Theoretical Models and Processes of Reading (6e éd., p. 1150-1181). International Reading Association. <https://doi.org/10.1177/002205741719700202>.

L'Homme, Marie-Claude \& Cormier, Monique. (2014). Dictionaries and the Digital Revolution: A Focus on Users and Lexical Databases. International Journal of Lexicography, 27(4), 331-340. <https://doi.org/10.1093/ijl/ecu023>.

MAULINI, Olivier \& PerRenoud, Philippe. (2005). La forme scolaire de l'éducation de base : tensions internes et évolutions. Dans O. Maulini \& C. Montandon (dir.), Les formes de l'éducation : variété et variations (p. 147-168). De Boeck Supérieur.

OLLIVIER, Christian. (2018). Littératie numérique et approche socio-interactionnelle pour l'enseignementapprentissage des langues. Conseil de l'Europe.

Penloup, Marie-Claude. (2012). Littératies numériques : quels enjeux pour la didactique de l'écriture-lecture ? Recherches en didactique des langues et des cultures, $9(2)$. <https://doi.org/ $10.4000 /$ rdlc.3900>.

POIBEAU, Thierry. (2019). Babel 2.0: Où va la traduction automatique? Odile Jacob.

SCRIBNER, Sylvia \& COLE, Michael. (2010). La littératie sans l'école : à la recherche des effets intellectuels de l'écriture. Langage et société, 133(3), 25-44. (Article original publié en 1978 sous le titre de « Literacy without Schooling: Testing for Intellectual Effects », Harvard Educational Review, 48(4), 448-461).

SELWYN, Neil. (2009). The Digital Native: Myth and Reality. Aslib Proceedings, 61(4), 364-479.

STREET, Brian. (1997). The Implications of the 'New Literacy Studies' for Literacy Education. English in Education, 31(3), 45-59. <https://doi.org/10.1111/j.1754-8845.1997.tb00133.x>.

VALIJÄRVI, Riitta-Liisa \& TARSOLY, Eszter. (2019). Translating Google Translate to the Language Classroom: Pitfalls and Possibilities. Practitioner Research in Higher Education, 12(1), 61-74.

\section{NOTES}

1. Les prénoms ont été modifiés à des fins d'anonymat.

2. Je distingue ici l'unité lexicale, qui peut être traduite par WordReference, du groupe nominal, qui inclut un déterminant. 


\section{RÉSUMÉS}

Les lycéens semblent s'être massivement appropriés les outils d'aide à la traduction dans le cadre des cours d'anglais, parfois malgré les préconisations des enseignants (Bourdais \& Guichon, 2020). L'étude de cas présentée ici vise à analyser, dans une démarche exploratoire, les pratiques effectives d'une lycéenne en contexte scolaire et à identifier les savoirs et savoir-faire mis en œuvre lors de la consultation de ces outils. Les pratiques numériques ont été observées par le biais de captures d'écran vidéos réalisées sur téléphone portable, dans le cadre d'activités de production écrite. L'analyse montre que la participante, qui suit un enseignement d'anglais de spécialité, a développé des compétences spécifiques aux outils consultés, mais qu'elle éprouve des difficultés à en rendre compte. D'autres études seraient nécessaires pour déterminer dans quelle mesure les compétences linguistiques des élèves influencent leurs pratiques ordinaires.

Highschool students seem to have seized online tools massively within the framework of English courses, sometimes despite the teachers' recommendations (Bourdais \& Guichon, 2020). The case study in question here aims to analyse the real practices of a highschool student in a school context, in an exploratory approach, and to identify the knowledge and the skills implemented when she uses these tools. Her digital practices were observed through video screenshots taken on her smartphone during writing activities. The analysis shows that the participant, who has specialised in English, has developed skills that are specific to the tools used, but that it is difficult for her to account for such skills. More research would be necessary to determine to what extent the students' linguistic skills influence their ordinary practices.

\section{INDEX}

Keywords : writing, highschool students, vernacular practices, online translator Mots-clés : production écrite, lycéens, pratiques vernaculaires, traducteur en ligne

\section{AUTEUR}

\section{AURÉLIE BOURDAIS}

Université Lumière Lyon 2, laboratoire ICAR 